\title{
Cortical spreading depression activates and upregulates MMP-9
}

\author{
Yasemin Gursoy-Ozdemir, ${ }^{1}$ Jianhua Qiu, ${ }^{1}$ Norihiro Matsuoka, ${ }^{1}$ Hayrunnisa Bolay, ${ }^{2}$ \\ Daniela Bermpohl, ${ }^{1}$ Hongwei Jin, ${ }^{1}$ Xiaoying Wang, ${ }^{3}$ Gary A. Rosenberg, ${ }^{4}$ \\ Eng H. Lo, ${ }^{3}$ and Michael A. Moskowitz ${ }^{1}$
}

1Stroke and Neurovascular Regulation Laboratory, Departments of Radiology and Neurology, Massachusetts General Hospital, Charlestown, Massachusetts,
USA. 2Faculty of Medicine Department of Neurology, Gazi University, Ankara, Turkey. ${ }^{3}$ Neuroprotection Research Laboratory, Departments of Radiology and
Neurology, Massachusetts General Hospital, Charlestown, Massachusetts, USA. ${ }^{4}$ Department of Neurology, University of New Mexico Health Sciences Center, Albuquerque, New Mexico, USA.

\begin{abstract}
Cortical spreading depression (CSD) is a propagating wave of neuronal and glial depolarization and has been implicated in disorders of neurovascular regulation such as stroke, head trauma, and migraine. In this study, we found that CSD alters blood-brain barrier (BBB) permeability by activating brain MMPs. Beginning at 3-6 hours, MMP-9 levels increased within cortex ipsilateral to the CSD, reaching a maximum at 24 hours and persisting for at least $\mathbf{4 8}$ hours. Gelatinolytic activity was detected earliest within the matrix of cortical blood vessels and later within neurons and pia arachnoid ( $\geq 3$ hours), particularly within piriform cortex; this activity was suppressed by injection of the metalloprotease inhibitor GM6001 or in vitro by the addition of a zinc chelator (1,10-phenanthroline). At 3-24 hours, immunoreactive laminin, endothelial barrier antigen, and zona occludens- 1 diminished in the ipsilateral cortex, suggesting that CSD altered proteins critical to the integrity of the BBB. At 3 hours after CSD, plasma protein leakage and brain edema developed contemporaneously. Albumin leakage was suppressed by the administration of GM6001. Protein leakage was not detected in MMP-9-null mice, implicating the MMP-9 isoform in barrier disruption. We conclude that intense neuronal and glial depolarization initiates a cascade that disrupts the BBB via an MMP-9-dependent mechanism.
\end{abstract}

\section{Introduction}

Cortical spreading depression (CSD) is a self-propagating depolarization associated with depressed neuronal bioelectrical activity and transient loss of membrane ionic gradients, and massive surges of extracellular potassium $(<50 \mathrm{mM})(1,2)$ and neurotransmitters as well as intracellular calcium (150 times the basal level). It was first described in 1944 by Leao (3). CSDs are observed in some clinical disorders like migraine, cerebrovascular diseases, head injury, epilepsy, and transient global amnesia, and often they are multiple (4-5). When combined with conditions imposing severe metabolic stress (e.g., hypoglycemia or ischemia), CSD exacerbates cellular injury and augments tissue damage, perhaps due to the energy burden of reestablishing ionic equilibrium in tissues that already have compromised blood flow and energy. CSD may also affect tissue outcome by causing profound transient hyperemia $(<200 \%$ of baseline) and long-lasting (up to 3 days) oligemia (60-90\% of baseline) (6). CSD is associated with a multitude of changes in immediate early genes, growth factors, and neurotransmitter and neuromodulatory systems, as well as inflammatory mediators such as interleukin-1 $\beta$ and tumor necrosis factor- $\alpha$ (7); however, it reportedly does not cause cell death in normal brains (8).

MMPs are a gene family of neutral proteases important in normal and abnormal brain function (9-12). They have been implicated in processes such as (a) opening of the blood-brain barrier (BBB) (13-15), (b) invasion of neural tissue by blood-derived

Nonstandard abbreviations used: blood-brain barrier (BBB); cortical spreading depression (CSD); endothelial barrier antigen (EBA); glial fibrillary acidic protein (GFAP); rat endothelial cell antigen (RECA); tissue plasminogen activator (tPA); zonula occludens-1 (ZO-1).

Conflict of interest: The authors have declared that no conflict of interest exists.

Citation for this article: J. Clin. Invest. 113:1447-1455 (2004).

doi:10.1172/JCI200421227. immune cells (16), (c) shedding of cytokines and cytokine receptors (12), and (d) direct cellular damage in diseases of the peripheral and central nervous systems (17). In addition, MMP activity contributes to interactions between cells and their matrix, allowing movement and shape changes important in processes such as development and neuronal plasticity. MMPs can be divided into five main groups based on protein structure: gelatinases (MMP-2 and MMP-9), collagenases (MMP-1, MMP-8, MMP-13, and MMP-18), stromelysins (MMP-3, MMP-10, and MMP-11), membrane-type MMPs (MMP-14, MMP-15, MMP-16, MMP-17, MMP-24, and MMP-25), and matrilysins (MMP-7 and MMP-26) (18).

Expression and activity of several MMPs have been well studied in brain during ischemia and inflammation (11). Asahi et al., using knockout technology, showed that MMP-9 has a central role in ischemic damage $(19,20)$. Knockout mice had smaller infarct volumes. Other studies have also shown that MMPs are important for $\mathrm{BBB}$ breakdown and for edema formation and leakage $(21,22)$. In CNS inflammation, such as in multiple sclerosis, MMPs may play an important role $(11,23)$.

MMPs disrupt the BBB, which comprises endothelial tight junctions, pericytes, astrocytic end feet, and basal lamina. The basal lamina contains extracellular matrix (ECM) molecules such as type IV collagen, laminin, and fibronectin, most of which are substrates for MMPs, especially MMP-2 and MMP-9 (12).

There is incomplete information regarding the effect of intense neuronal and glial depolarization, as occurs during CSD, on matrix and blood vessel function, and this has been speculated to be of importance in neurovascular regulation (24). In this report we studied the relationship between CSD, MMP activation, and BBB changes. We observed that CSD causes prolonged MMP-9 activity that leads to breakdown of the BBB, edema formation, and vascular leakage that can be suppressed by an MMP inhibitor. 


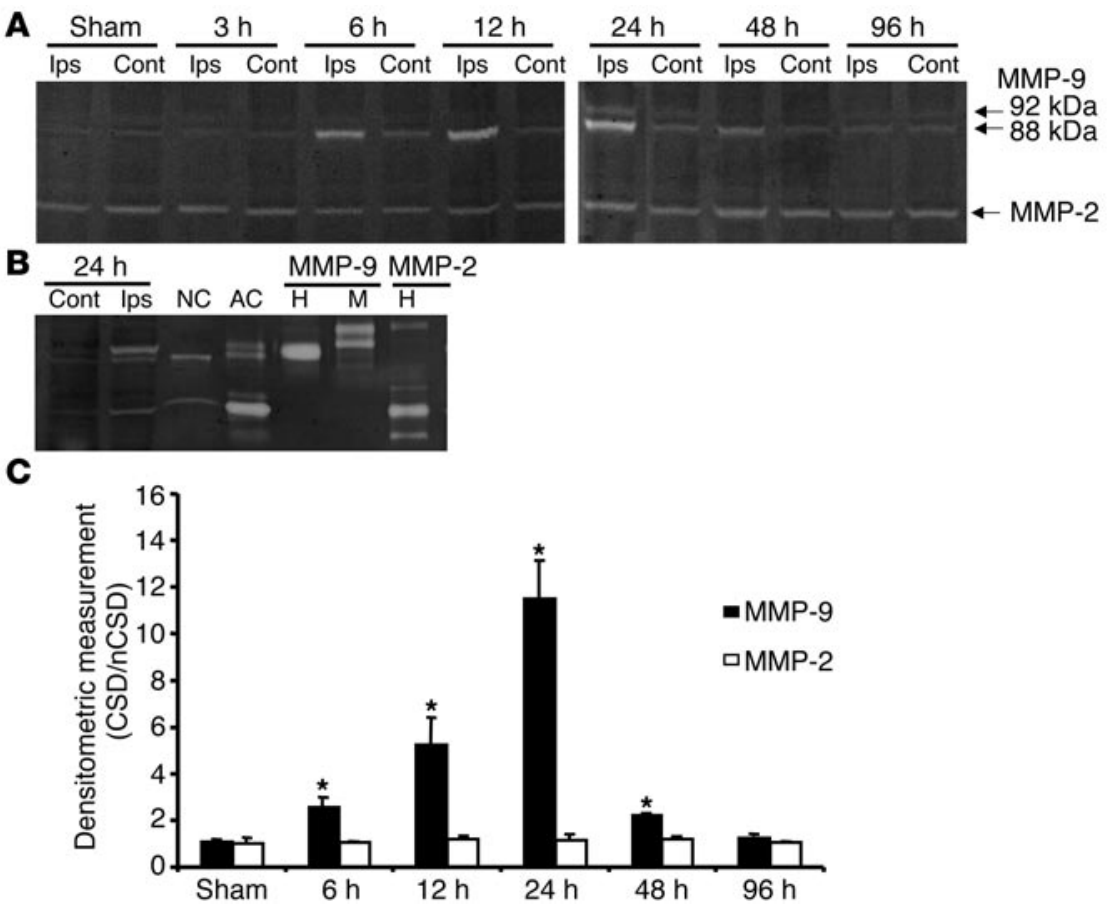

\section{Figure 1}

CSD caused time-dependent increases in MMP-9 levels, as assessed by gelatin gel zymography. Unilateral CSD was elicited by pinprick in the frontal cortex as described (see Methods). (A) MMP-9 gelatinolytic activity was consistently higher on the CSD side from 6 to 48 hours and returned to basal levels by 96 hours. The molecular weights of both bands corresponded to those of rat MMP-9 (92 kDa and $88 \mathrm{kDa}$ ). (B) Comparison of MMP-9 responses induced by CSD versus MMP-9 in conditioned media from rat cortical neuron (NC) and astrocyte (AC) cell cultures incubated with $100 \mathrm{nM}$ phorbol myristate acetate (PMA) for 24 hours. $92 \mathrm{kDa}$ recombinant human MMP-9 $(\mathrm{H})$ and $105 \mathrm{kDa}$ recombinant mouse MMP-9 (M) standards were also loaded as molecular weight markers. MMP-9 bands induced by CSD at 24 hours matched those obtained from neuron and astrocyte cultures. Ips, ipsilateral (CSD); Cont, contralateral (non-CSD). (C) Densitometric measurements confirm the increase in MMP-9 induced by CSD. Constitutive MMP-2 expression and activity did not change after CSD and were visualized in sham as well. nCSD, non-CSD. ${ }^{*} P<0.05$ compared with the contralateral side.

\section{Results}

Compared with results in animals subjected to anesthesia and craniotomy but without CSD induction (sham) control, CSD increased MMP-9 levels within cortical homogenates, as shown by gelatin gel zymography (Figure 1, A and B). Bands corresponding to MMP-2 gelatinolytic activity were visible in "control" animals but did not change after CSD $(n=6$ per group, except $n=2$ at 48 and 96 hours) (Figure 1, A and C). MMP-9 levels increased in two of six animals at 3 hours (not shown), was consistently higher between 6 and 48 hours $(P<0.05)$, and disappeared by 96 hours. All samples at 24 hours exhibited two MMP-9 bands (88 and 92 $\mathrm{kDa}$ ) whose molecular weights corresponded to gelatin proteases that appeared on gel zymograms of conditioned media from primary rat cortical neurons and astrocytes incubated with $100 \mathrm{nM}$ phorbol myristate acetate (PMA) for 24 hours (Figure 1B). We observed MMP-9 mRNA in sham animals, suggesting the possibility that MMP-9 is constitutively expressed and upregulated in cortex after CSD. Induced MMP-9 mRNA expression was found by RT-PCR (Figure $2 ; n=3$ per time point) beginning 1 hour after CSD, consistent with transcriptional changes and other data indicating new MMP-9 protein synthesis (Figure 1A).

To rule out the possibility that trauma (i.e., needle stab), not the effect of CSD, increased MMP levels, we applied the NMDA receptor blocker and CSD inhibitor MK-801 topically 15 minutes before CSD. MK-801 blocked CSDs and also inhibited the development of increased MMP-9 level at 12 hours in tissues remote from the needle stab (Figure 3A; $n=3$ ). This result clearly showed that MMP-9 activity is related to CSD propagation and is less likely to be related to the trauma used to initiate it.

We next tested whether administration of an MMP inhibitor systemically reduced CSD-induced gelatin-cleaving activity. We observed a marked reduction in MMP-9 level in cortical homogenates (Figure 3B; $n=3$ ). These data are consistent with a previous report showing that MMP zymographic activity decreas- es after administration of a nonselective MMP inhibitor in vivo (25). They strengthen the observation that CSD increased MMP activity and that the evidence obtained by gelatin zymography was specific for MMP.

To determine the spatial location of MMP-9 activity after CSD, we examined cleavage of the fluorogenic substrate DQ gelatin in tissue sections (Figure 4, A and B). A single CSD increased gelatinolytic activity in blood vessels examined 15-30 minutes after CSD (Figure 4F) (48 \pm 6 versus $77 \pm 8$ positive blood vessels on the contralateral and CSD sides, respectively; $P<0.05)$. At 3 hours, gelatin-cleaving activity was present in cells (laminae II-IV; $n=4$ ) within sections from piriform to occipital cortex, but was particularly prominent within piriform cortex (Figure 4). At 6 hours, 12 hours (data not shown; $n=4$ ), and 24 hours (Figure 4B; $n=4$ ), fluorescent product was found in cells, pia arachnoid, and blood vessels (Figure 4, C-E). Activity in the hippocampus was robust bilaterally in both sham and experimental groups, consistent with the constitutive MMP expression reported previously (26), and this activity did not appear to increase after CSD. Nearly all fluorogenic cleaving activity was blocked by preincubation of tissue sections with a zinc chelator (1,10-phenanthrolin) for 15 minutes (not

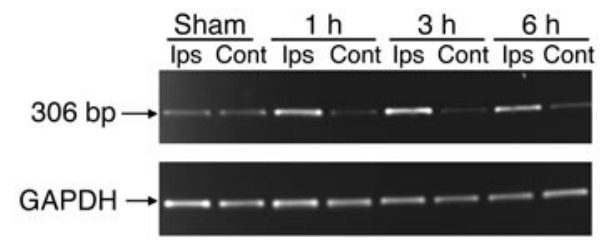

\section{Figure 2}

CSD upregulated MMP-9 mRNA in piriform cortex at 1, 3, and 6 hours. The housekeeping gene GAPDH is shown for comparison. MMP-9 mRNA was constitutively expressed in both brain cortices from control and contralateral to CSD. 


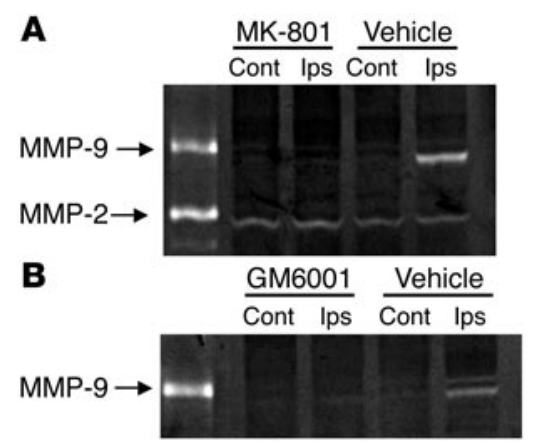

\section{Figure 3}

The NMDA receptor blocker MK-801 blocked CSD and suppressed MMP-9 activity. (A) Topical MK-801 was administered 15 minutes before CSD. No change in MMP-2 expression was detected. These results suggest that MMP- 9 activity is related to CSD but not to the needle stab $(n=3)$. (B) The nonselective metalloprotease inhibitor GM6001 was administered intraperitoneally 1 and 4 hours after CSD (65 mg/kg for each). A marked reduction in MMP-9 activity was found by gel zymography 12 hours later (two of three samples showed decreased or no MMP level). These results indicate that CSD increased MMP activity in vivo.

shown), confirming the specificity of this reaction. After injection of a metalloprotease inhibitor, GM6001, in situ gelatinolytic activity decreased in neurons, meninges, and penetrating blood vessels examined 12 hours later (data not shown).

Gelatinolytic activity was mostly found in large penetrating pial vessels (>20 $\mu \mathrm{m}$ in diameter) as well as within tissue membranes in the pia and arachnoid. To identify the cell types expressing gelatin cleaving activity, we stained sequentially single sections for both gelatin cleaving activity and expression of cell-specific antigens (Figure $5 ; n=5$ ). Figure 5A shows that most gelatinolytic activity was present within neurons and was visualized in the walls of blood vessels. Only infrequent colocalization was found between glial fibrillary acidic protein (GFAP), an astrocytic marker, and gelatin cleaving activity. A particularly prominent example is shown in Figure 5B. Colocalization was also uncommon in endothelial cells (Figure 5C) and smooth muscle cells (Figure 5D). The results did not differ when optical sections $1.8-5.1 \mu \mathrm{m}$ in thickness were examined by laser-scanning confocal microscopy. Hence, most of

\section{Figure 4}

CSD increased in situ gelatinolytic activity in cortex ipsilateral to CSD. (A-E) Activity appeared as green fluorescent product and developed after incubation of coronal sections (10 $\mu \mathrm{m}$ in thickness) with the fluorogenic substrate DQ gelatin. Increased gelatinolytic activity was detected at 3 hours (A) and 24 hours (B). The image in $\mathbf{C}$ represents a higher magnification of the boxed area in $\mathbf{A}$ (scale bar: $100 \mu \mathrm{m}$ ). $\mathbf{C}$ shows gelatinolytic activity in penetrating blood vessels (single arrow) and in pia arachnoid (opposing arrows). (D and E) Activity is visualized in a blood vessel (arrow) cut in cross-section (D) and in a vessel (arrow) showing corrugations (E). Scale bars: $20 \mu \mathrm{m}$. Gelatinolytic activity was inhibited by preincubation with 1,10-phenanthroline (a nonselective zinc chelator) (data not shown). (F) In situ gelatinolytic activity was observed around blood vessels as early as 30 minutes to 1 hour after a single CSD, and the number of labeled vessels was significantly higher on the CSD side than on the non-CSD side or in sham-treated animals $\left({ }^{\star} P<0.05\right.$ compared with non-CSD side or sham cortices for right and left hemispheres). the cleaving activity of the fluorogenic substrate was present within the vessel matrix.

MMPs, especially MMP-9, cleave basement membrane proteins and disrupt the BBB. Using Western blot $(n=6)$ and immunohistochemistry $(n=4)$, we found that CSD caused BBB disruption and a decrease in the MMP substrate protein laminin (Figure 6, A and C). Endothelial barrier antigen (EBA) was also reduced (Figure $6 \mathrm{C} ; n=4$ ). As anticipated, zonula occludens-1 (ZO-1), a tight junction protein, colocalized with rat endothelial cell marker (RECA). However, after CSD this decreased (Figure 6B; $n=4)$. These results suggest that CSD may affect proteins important for BBB function.

The vital dye Evans blue binds tightly to albumin, and this complex is excluded from crossing the intact BBB. We therefore examined leakage of Evans blue in the brain after CSD and detected this tracer in increased amounts in brain homogenates within 3-24 hours, as compared with leakage in the sham group and on the non-CSD side (Figure 7A; $n=5$ per time point). Evans blue was prominently visualized surrounding blood vessels. Vascular leakage of Evans blue was found on both sides (CSD and non-CSD) at 12 hours, but the number of staining vessels was higher on the CSD side (CSD, $19 \pm 1.5$, vs. non-CSD, $7 \pm 0.6$; Figure 7B). Differences between the two sides were also found by quantitative flourometric measurements (Figure 7A). Evans blue leakage was long lasting and was observed when this tracer was injected 21 hours after CSD (Figure $7 \mathrm{C}$ ). Furthermore, edema developed within cortical gray matter with a time course consistent with albumin leakage (Figure 7D). The magnitude of the edema in cortical grey matter (approximately $3.0-4.6 \%$ above control) was less than the $6-9 \%$ reported in cortex after ischemia $(27,28)$.

To address the role of increased MMP-9 activity on CSDinduced tracer leakage, we administered the metalloprotease inhibitor GM6001 and found that it completely blocked the presence of Evans blue in brain 12 hours after CSD (Figure 8; $\mathrm{CSD} /$ non-CSD ratio, $2.2 \pm 0.3$ versus $0.95 \pm 0.18$ after vehicle and drug treatment, respectively; $n=5$ per group; $P<0.05$ ) but did
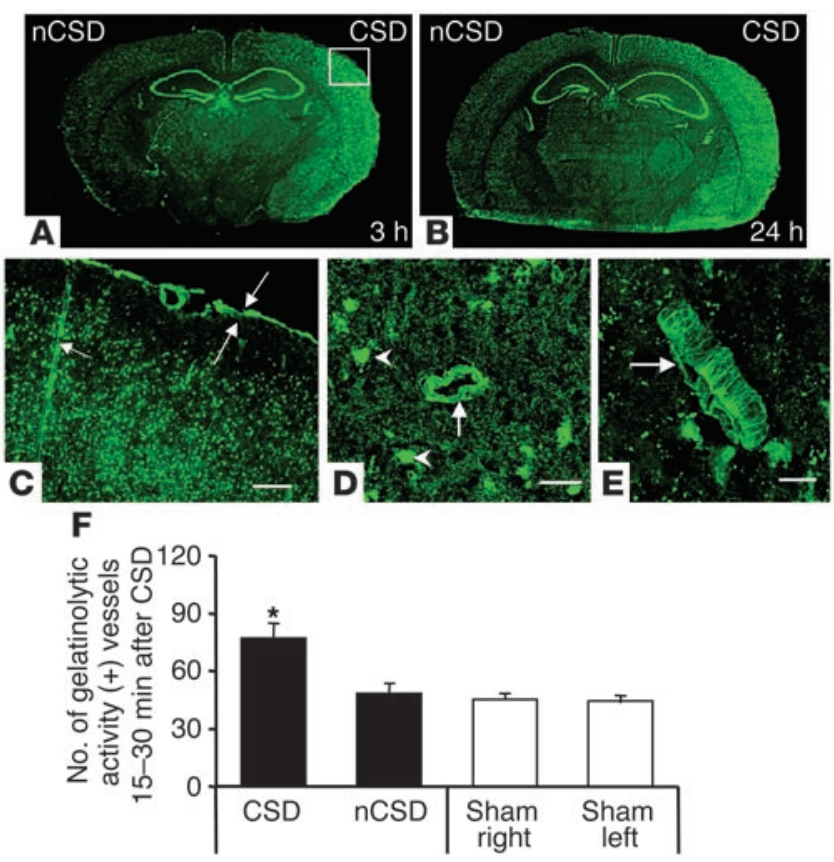
not affect the electrophysiological properties of CSD. Preliminary results using BB94, a nonselective metalloprotease inhibitor, confirmed these findings (data not shown).

As a second approach, we reasoned that if MMP-9 activation were essential for CSD-induced albumin leakage, than extravasated vital dye would be suppressed or absent in MMP-9-null mice. Tracer leakage was significantly decreased at 6 hours in $M M P-9$ knockout mice compared with age-matched control mice of similar background strain (CD-1) (Figure 8; CSD/non-CSD ratio, $1.1 \pm 0.1$ versus $2.6 \pm 0.3$ for MMP-9-null mice and the wild-type strain, respectively; $n=5 ; P<0.05)$. The electrophysiological properties of CSD did not differ between wild-type and mutant mice. These data implicate MMP-9 in the BBB disruption that follows CSD.

\section{Discussion}

We report the novel finding that CSD activates and upregulates MMPs and promotes sustained changes in vascular permeability. MMP-9 activation was detected as early as 15-30 minutes by in situ zymography and later by gelatin zymography. At 3 hours, MMP activity appeared within neurons and pia arachnoidal mem- branes. We also detected Evans blue leakage and edema in cortical tissues at this time point. MMP-9 activation was long lasting, observed 48 hours after induction. MMP activity was first observed within penetrating cortical arterioles, but did not colocalize within endothelium or smooth muscle cells; it appeared prominently within the vessel matrix. The rapid onset of MMP-like activity suggested to us that blood vessels constitutively express MMPs, perhaps synthesized within and released as a consequence of CSD from the endothelium $(29,30)$ or smooth muscle. In fact, constitutive MMP-9 was reportedly expressed in hippocampal blood vessels $(31,32)$. It is well known that vascular smooth muscle cells synthesize and release MMP-9, particularly when exposed to such stimuli as mechanical injury, PMA, fetal calf serum, thrombin, and interleukin-1 $\alpha$, and concomitant with advanced age (33-36).

At 3 hours after CSD, most of the in situ gelatinolytic activity was localized within neurons. Consistent with our results, others have shown that MMP-9 and MMP-2 were visualized within the granular cell layer and Bergman glia in adult cerebellum (37). MMP activity is important for synaptic and extracellular reorganization or neurotoxicity (in ischemia and trauma) and it increases within hippocampal neurons from a high basal level after kainite-
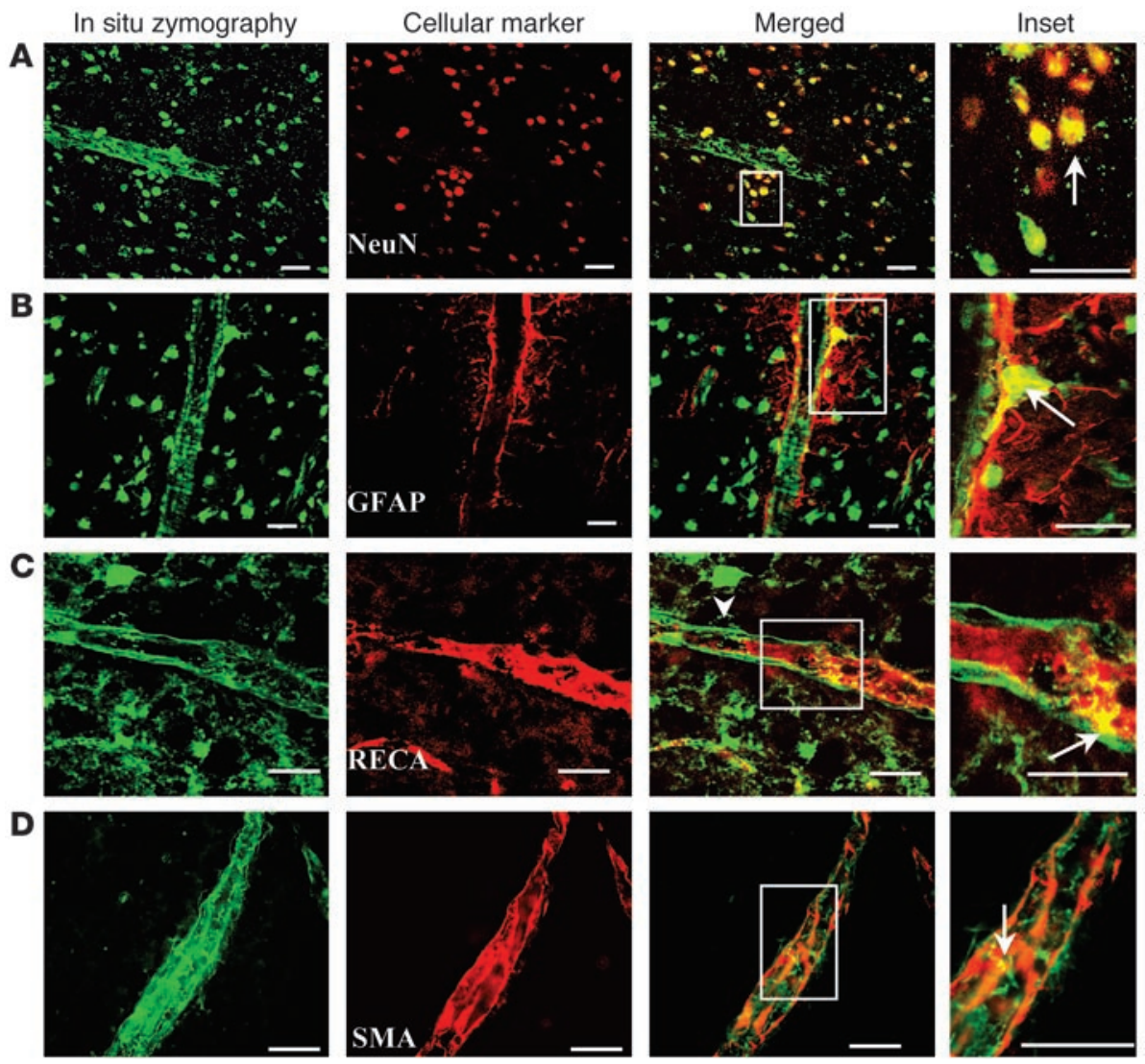

Figure 5

CSD increased gelatinolytic activity within the vascular matrix. (A-D) Distribution of DQ gelatin-cleaving activity (in situ zymography) was compared on single sections, with the locations of cells identified by staining with specific cell markers: astrocytes, GFAP; neurons, NeuN; endothelial cells, RECA; smooth muscle cells, SMA. Cell markers were visualized by Cy-3 conjugated secondary antibody and were stained red in color. Boxed areas from merged confocal images (Merged column) are shown at higher magnification in the far right column (Inset). Gelatin cleaving activity was prominent in the walls of blood vessels and within surrounding neurons. Cleaving activity was rarely found colocalized with GFAPpositive cells. The figure shows one example. Infrequent activity was detected in endothelial cells and smooth muscle cells but predominantly within the vascular matrix. Sections were obtained 12 hours after CSD. Arrows point to areas showing colocalization. Scale bars: $20 \mu \mathrm{m}$. 
A

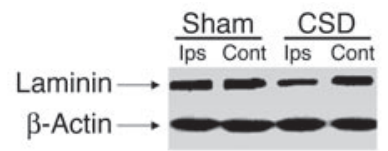

B

RECA
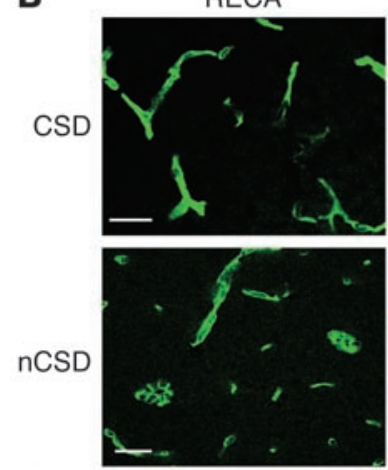

C CSD

EBA
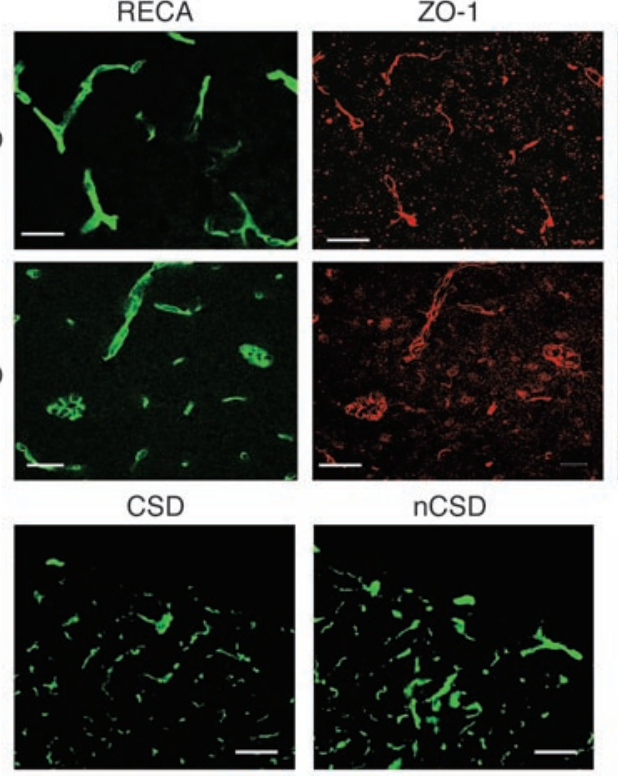

Laminin

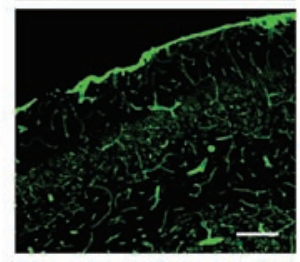

$\mathrm{nCSD}$

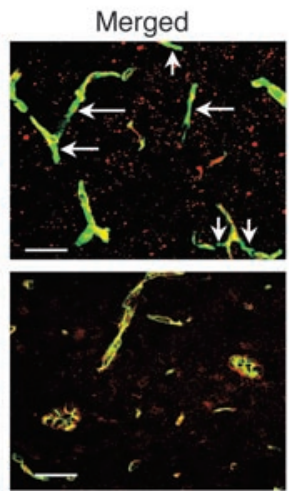

Pt?

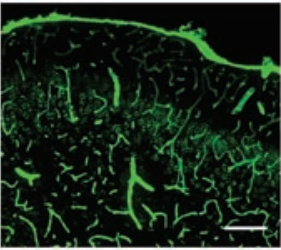

induced seizure (26). Vecil et al. showed that neurons "expressed" MMP-9 and MMP-2 gelatin cleaving activity in vitro under basal conditions and that inflammatory stimuli such as cytokines like interleukin-1 $\beta$ upregulate their expression (38). The consequences of neuronal MMP expression are not known. Release into the extracellular space after CSD may contribute to vascular changes we observed after CSD.

Oxygen radicals, nitric oxide (NO), and proteases have been implicated in MMP activation. During CSD, proinflammatory cytokines (6) and NO increase within brain tissue $(39,40)$. NO synthesis is rapid and sustains for at least 1 hour (39). NO reportedly $S$-nitrosylates a critical cysteine residue at the catalytic pocket by displacing zinc (41). Other oxygen radicals such as superoxide have been implicated as well $(42,43)$. Proteases such as stromelysin- 1 and plasmin have been associated with MMP-9 activation. The method of activation of the inactive form of MMP-9 will need to be studied further.

CSD stimulates c-fos, tumor necrosis factor- $\alpha$, interleukin- $1 \beta$ production (7), and nuclear translocation of the NF-кB subunit p65 (our unpublished data). All four bind the promoter region of MMP-9 $(9,10)$. In accordance with these observations, we found MMP-9 mRNA upregulation between 1 and 6 hours after CSD. Furthermore, MMP-9 mRNA upregulation was reported after potassium chloride-induced CSD, confirming a critical link between depolarization and MMP gene upregulation (44).

MMPs cleave a broad range of substrates, including components of basal lamina like laminin, type IV collagen, and brain

\section{Figure 6}

CSD altered the immunostaining of laminin, ZO-1, and EBA within the blood vessel wall. (A-C) Laminin (approximate molecular weight, $200 \mathrm{kDa}$ ), a basement membrane protein, was reduced ipsilateral to CSD on immunoblots (at 12 hours) compared with that of the sham group and compared with that of the contralateral side. Similarly, CSD decreased the immunostaining of laminin in blood vessels (C), indicating that CSD alters the antigenicity of an integral basement membrane protein (scale bars: $100 \mu \mathrm{m}$ ). (B) These images show colocalization of the tight junction protein ZO-1 with RECA and show that CSD decreases the extent of this colocalization (colocalization coefficient, $0.21 \pm 0.03$ for CSD versus $0.55 \pm 0.05$ for control; $P<0.05$ ). Compared with the image of the opposite side (nCSD), the merged image after CSD shows large segments of blood vessels with green fluorescence only (arrows; scale bars: $20 \mu \mathrm{m})$. (C) EBA immunostaining was also reduced in cortical blood vessels after CSD. This decrease often indicates impaired BBB function (scale bars: $100 \mu \mathrm{m}$ ).

ECM components like elastin, fibronectin, tenascin, and collagens. After ischemia or trauma, laminin, type IV collagen, and the tight junction protein $\mathrm{ZO}-1$ are reduced (45). We observed a decrease in laminin immunoreactivity 12 hours after CSD, as well as a decrease in EBA and ZO-1. In vivo targeting of EBA (with anti-EBA) leads to opening of the BBB apparently via paracellular and transcellular routes, although the exact role and identity of EBA is not known (46). After traumatic brain injury, EBA decreases in the zone of cortical contusion (47) and in ischemic brain (48). However, we cannot state based on existing data whether EBA, laminin, or ZO-1 is directly cleaved by MMP in this model. However, the loss of ZO-1, a critical intracellular tight junction protein (20), and loss of laminin immunoreactivity (49) are consistent with BBB dysfunction. Kakinuma et al. found that laminin production is reduced in astrocytes when BBB permeability is enhanced in response to cold injury (49). In accordance with these data, we observed increased albumin and brain water content contemporaneously with loss of ZO-1 and laminin. Evans blue leakage (and, we presume, increased brain water content) was MMP dependent, indicating an effect of CSD and MMP-9 on BBB function. The relative contributions of CSD-induced vascular and neuronal MMP activity to changes in brain vascular permeability were not determined.

To prove that BBB dysfunction develops as a specific consequence of MMP-9 activation, we first tested an MMP inhibitor and showed that leakage of albumin and MMP activity were blocked. Second, we applied a zinc chelator that blocked most of the gelatin-cleaving activity in situ, indicating that proteases that require zinc for their function (e.g., MMP-9) caused gelatin cleavage. As a third approach, we used genetically engineered mice with a deletion in the MMP-9 gene to show that MMP-9 was the protease essential for leakage. MMP-9 knockout mice showed decreased leakage. These findings are consistent with published data showing that MMP-9-null mice have less BBB disruption and leakage after experimental ischemia and trauma, suggesting that mechanisms of BBB disruption between ischemia, trauma and CSD overlap $(19,20)$. We cannot rule out a possible contribution of tissue 
plasminogen activator ( $\mathrm{tPA}$ ) to MMP activation during early time points. It is known that tPA is released after depolarization by calcium-mediated mechanisms in neurons in vitro (50), and tPA can upregulate MMP-9 (30), as can other MMP subtypes. Consistent with this idea, we detected MMP-8 gene upregulation at 24 hours after CSD (our unpublished data).

There is abundant experimental evidence that repetitive spreading depressions contribute to the evolution of infarcts in peri-infarct tissue in rats and cats (51-54). BBB disruption may sometimes accompany stroke, trauma, and migraine, and one recent report indicates that migraineurs with aura have a high prevalence of cerebellar infarcts if they experience one or more attack(s) per month (55). Our data suggest the possibility that peri-infarct CSD may open the $\mathrm{BBB}$ and promote sustained leakage of serum proteins by MMP-dependent mechanisms. Barrier disruption may contribute to cell death and an increase in infarct volume in compromised brain. Hence, CSD may augment MMP upregulation and vascular permeability changes in migraine, stroke, and trauma and may possibly contribute to susceptibility to cerebral hemorrhage $(54,56,57)$.

\section{Methods}

CSD. The CSD model was used as described previously $(24,58)$ and all experiments were performed following an institutionally approved protocol in accordance with The National Institutes of Health Guide for the Care and Use of Laboratory Animals under institutional guidelines. Briefly, male Sprague Dawley rats (250-300 g body weight) or male mice (MMP-9-null mice and age-matched CD1 mice; $25 \mathrm{~g}$ body weight) were anesthetized via intraperitoneal injection of sodium pentobarbital $(60 \mathrm{mg} / \mathrm{kg})$ and were placed in a stereotaxic frame. The dura mater was opened carefully bilaterally. Recording electrodes were placed symmetrically over both hemispheres (frontal: 1-3 mm anterior and 2-4 $\mathrm{mm}$ lateral to the bregma for rats; $1-2 \mathrm{~mm}$ anterior and $1-3 \mathrm{~mm}$ lateral to the bregma for mice) unless noted otherwise. In every experiment, a sham group was prepared by making a bilateral

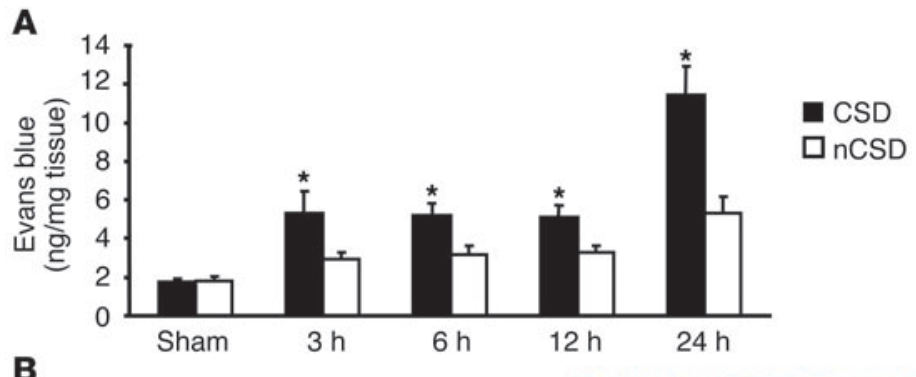

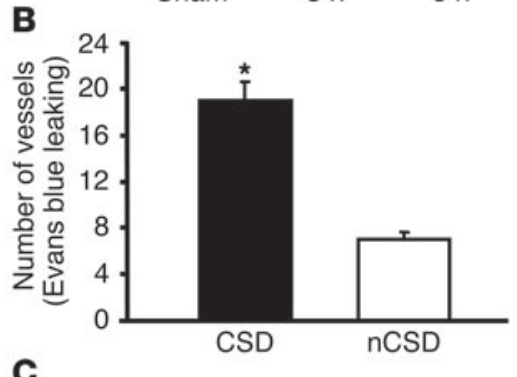

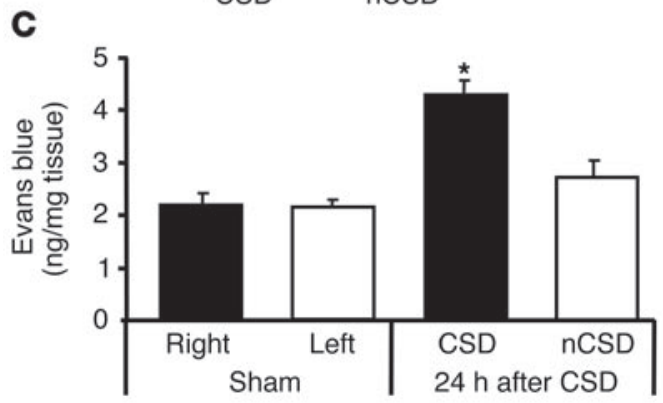

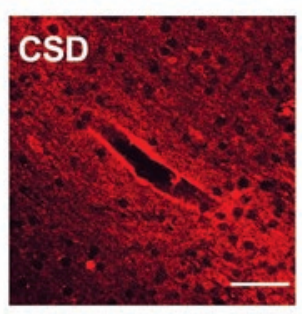

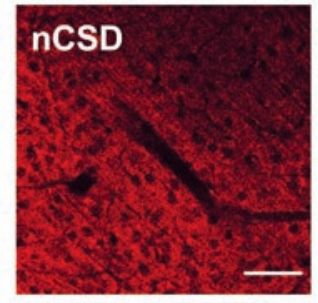

D

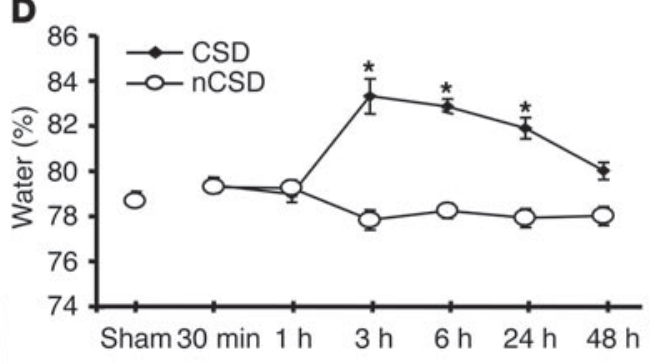

\section{Figure 7}

CSD causes Evans blue leakage and edema within cerebral cortex. (A) Evans blue was injected 15 minutes prior to the onset of CSD. The vital dye (Evans blue) was extracted from cortical tissues at the indicated time points. There was a statistically significant difference $\left({ }^{*}\right.$, see below) between leakage into the CSD cortex and that of the sham group or the contralateral side. (B) Plasma proteins leak from cortical blood vessels after CSD. The number of vessels (>10 $\mu \mathrm{m}$ in diameter) in which leakage was detected on both CSD and non-CSD (contralateral) cortex are shown for six animals $(P<0.05)$. Confocal images show one vessel leaking Evans blue on the CSD side and a non-leaking vessel on the non-CSD side (scale bars: $100 \mu \mathrm{m}$ ). (C) Evans blue leakage was long lasting. When injected at 21 hours after CSD, vital dye was detected in CSD cortex 3 hours later and it was higher than that of sham cortex and the non-CSD side $(n=5 ; P<0.05)$. (D) CSD caused a time-dependent increase in edema, as expressed by percentage water content (wet-dry/wet) brain weights. At 3, 6, and 24 hours, edema measured in piriform cortex increased on the CSD side compared with that of the non-CSD side and sham group. Asterisk denotes significant difference compared with contralateral cortex or to the sham group in $\mathbf{A}-\mathbf{D} ; P<0.05$. 


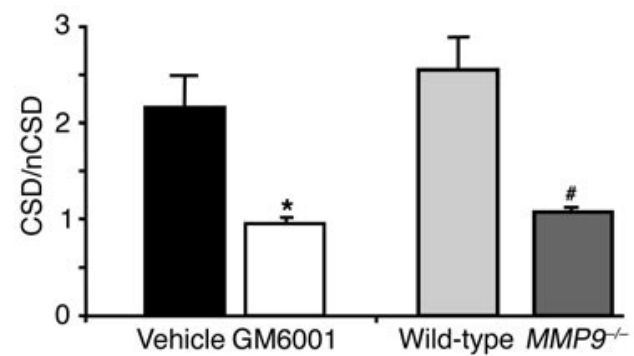

Figure 8

CSD-induced plasma protein leakage was suppressed after MMP inhibition or after CSD in mice lacking MMP-9 gene expression. Evans blue leakage is blocked by the nonselective metalloprotease inhibitor GM6001 (65 mg/kg intraperitoneally, given 1 hour before and 4 hours after CSD) when examined at 12 hours, compared with that of vehicletreated animals ( $n=5$ per group; ${ }^{*} P<0.05$ vs. vehicle). A decrease in leakage 6 hours after CSD was detected in mice lacking MMP-9 gene expression compared with that of the same age-matched mice of similar background (CD-1) ( $n=5$ per group; ${ }^{\#} P<0.05$ vs. wild-type strain), although CSD itself did not differ between strains. Data are expressed as a ratio of Evans blue leakage in homogenates from both sides.

craniotomy and opening the dura mater in anesthetized animals. No electrodes were inserted. In the experimental groups, cortical steady potentials and electrocorticograms were recorded by singlebarrel glass microelectrodes inserted $900 \mu \mathrm{m}$ into the cortex for rat and $400 \mu \mathrm{m}$ into the cortex for mice or placed on the surface to prevent CSD during electrode insertion. CSD was initiated by pinprick with a 30-gauge needle. Rectal temperature was monitored continuously and body temperature was kept at $37 \pm 0.5^{\circ} \mathrm{C}$ until the animals awoke from anesthesia. A three-CSD paradigm was routinely used unless noted otherwise. CSDs and their electrophysiological characteristics were monitored carefully.

Gelatin gel zymography. Tissue samples were homogenized (and immunoprecipitates were resuspended) in "working buffer" (50 $\mathrm{mM}$ Tris-HCl, pH 7.6, $150 \mathrm{mM} \mathrm{NaCl}, 5 \mathrm{mM} \mathrm{CaCl}_{2}, 0.05 \%$ BRI-35, and $0.02 \% \mathrm{NaN}_{3}$ ). Proteins $(25 \mu \mathrm{g})$ were separated by electrophoresis through $10 \%$ polyacrylamide zymogram gels containing gelatin (Invitrogen, Carlsbad, California, USA) under nondenaturating and nonreducing conditions. After washes in $2.5 \%$ Triton X-100 to remove SDS and then in $50 \mathrm{mM}$ Tris- $\mathrm{HCl}, \mathrm{pH} 7.5$, the gels were incubated overnight in developing buffer $(50 \mathrm{mM}$ Tris- $\mathrm{HCl}, \mathrm{pH} 7.5$, $10 \mathrm{mM} \mathrm{CaCl}_{2}, 1 \mu \mathrm{M} \mathrm{ZnCl}_{2}$, and $0.05 \% \mathrm{BRIJ}-35$ ) at $37^{\circ} \mathrm{C}$. Gels were stained with $0.25 \%$ Coomassie blue G-250 in 20\% methanol and $10 \%$ acetic acid, then were washed in solution with $20 \%$ methanol and $10 \%$ acetic acid before being photographed and analyzed by densitometry (MCID Elite, St. Catharines, Ontario, Canada).

In situ zymography and double labeling with fluorescent probes. In situ gelatinolytic activity was performed on frozen brain sections 10 $\mu \mathrm{m}$ in thickness using a commercial kit (EnzCheck Gelatinase Assay Kit; Molecular Probes, Eugene, Oregon, USA). Sections were incubated with DQ gelatin conjugate, a fluorogenic substrate, at $37^{\circ} \mathrm{C}$ overnight and then, after being washed, were fixed in $4 \%$ paraformaldehyde in phosphate-buffered saline (PBS). Cleavage of DQ gelatin by MMPs resulted in a green fluorescent product (wavelengths: excitation, $495 \mathrm{~nm}$; emission, $515 \mathrm{~nm}$ ). Homologous brain regions were compared. Some tissue sections were incubated with the zinc chelator 1,10-phenanthroline (1 $\mathrm{mM}$ in DMSO; Molecular Probes), a nonspecific inhibitor of MMP activity.
After in situ gelatinolytic activity was assessed, some fixed tissue sections were incubated with Cy3-conjugated antibodies specific for neurons (NeuN; 1:200 dilution; Chemicon, Temecula, California, USA), astrocytes (GFAP; 1:200 dilution; Sigma-Aldrich, St. Louis, Missouri, USA), endothelium (RECA; 1:200 dilution; Serotec, Raleigh, North Carolina, USA), and vascular smooth muscle (SMA; 1:200 dilution; Sigma-Aldrich). Sections mounted using an anti-fade kit (SlowFade; Molecular Probes) were examined with a Zeiss LSM-Pascal confocal laser-scanning microscope (Oberkochen, Germany) and/or a fluorescence microscope. Single optical sections (1.8-5.1 $\mu \mathrm{m}$ in thickness) were imaged.

Quantification of vessels positive for in situ zymography. Sections were obtained 15-30 minutes after one CSD. Positively labeled vessels on both the CSD and non-CSD sides were counted manually over all cortical lamina (magnification, $\times 200$ ) in four consecutive sections obtained about $4 \mathrm{~mm}$ posterior to the frontal pole and passing through the hippocampus. The raters were unaware of the experimental protocol used for each sample.

Immunobistochemistry. After an overdose of pentobarbital, animals were perfused intracardially with heparinized saline $(50 \mathrm{ml}$ for rat and $20 \mathrm{ml}$ for mice) followed by $4 \%$ paraformaldehyde in PBS. Brains were post-fixed for 24 hours with the same fixative, then were cryoprotected in $30 \%$ sucrose and sectioned at 40 $\mu \mathrm{m}$. For immunohistochemical staining, sections were washed in PBS and incubated in 10\% donkey serum in PBS containing $0.35 \%$ Triton X-100 for 30 minutes. Then they were incubated with primary antibodies in $2 \%$ donkey serum overnight at $4^{\circ} \mathrm{C}$. Antibodies against EBA (1:200 dilution; Stenberger Monoclonals Inc., Lutherville, Maryland, USA), laminin (1:500 dilution; SigmaAldrich), and ZO-1 (1:100 dilution; Zymed, South San Francisco, California, USA) were used. Primary antibody omission, incubations with either blocking solution or PBS, and comparison with Western blot analysis were carried out to test the specificity of the immunoreactivity observed. After being washed, sections were incubated at room temperature for 3 hours in $2 \%$ donkey serum with FITC-labeled secondary antibodies (1:200 dilution). Sections were then washed and mounted using an anti-fade kit (SlowFade; Molecular Probes) and were examined with a Zeiss LSM-Pascal confocal laser-scanning microscope and/or a fluorescence microscope. Single optical sections (1.8-5.1 $\mu \mathrm{m}$ in thickness) were collected and "pseudocolored" according to their original fluorochromes. Blood vessels (full thickness) were imaged. Blood vessels (full thickness) were examined by Z-stack imaging (LSM Pascal, Zeiss, Oberkochen, Germany).

Quantification of ZO-1 and RECA. ZO-1 immunostaining was performed as described in Methods. Sections were obtained 12 hours after three CSDs. After being washed in PBS, sections were incubated for 3 hours at room temperature in $2 \%$ donkey serum with RECA (1:200 dilution; Serotec). A Cy3-labeled secondary antibody was used for ZO-1 labeling and an FITC-labeled one was used for RECA labeling. Double-labeled sections (three per each animal) passing through hippocampus were analyzed with a Zeiss LSMPascal confocal laser-scanning microscope. The cortex of each section was divided into ten regions of interest on both the CSD and non-CSD sides and the colocalization coefficient was calculated per area. The mean of three sections per animal was obtained; values are expressed as mean + SEM.

Western blot analysis. Tissues were homogenized in radioimmunoprecipitation buffer $(20 \mathrm{mM}$ Tris-HCl, $\mathrm{pH} 7.5,50 \mathrm{mM} \mathrm{NaCl}, 1 \mathrm{mM}$ EDTA, $0.5 \%$ NP-40, $0.5 \%$ SDS, and $0.5 \%$ deoxycholic acid), PMSF 
(0.1 mM), and proteinase inhibitor cocktail (Sigma-Aldrich). After protein measurement and polyacrylamide gel electrophoresis, the proteins were transferred to PVDF membranes and incubated with laminin antibody (1:500 dilution; Sigma-Aldrich). After being washed in Tris-buffered saline with $0.1 \%$ Tween-20 (SigmaAldrich), membranes were incubated in secondary horseradish peroxidase-labeled antibody for 1 hour. Then proteins were visualized using the enhanced chemiluminescence procedure (Amersham Biosciences, Piscataway, New Jersey, USA). $\beta$-Actin was used as an internal standard. Densitometry was performed using MCID Elite software.

$R T-P C R$. Total RNA was isolated from CSD and non-CSD brain tissue using Trizol and reverse transcription was performed using SuperScript II (Invitrogen, Carlsbad, California, USA). A first-strand synthesis system (Invitrogen) was used according to the manufacturer's recommendations. The following primers were used: rat MMP-9, 5'-AGATGTGGGTGTACACAGGC-3' and 5'-TTCACCCGGTTGTGGAAACT-3'; rat GAPDH, 5'-TTAAGGGCATCCTGAGCTACACT- $3^{\prime}$ and $5^{\prime}$-TTACTCCTTGGAGGCCATGTAGG-3'.

Pharmacological studies. The broad-spectrum metalloprotease inhibitor GM6001 (65 mg/kg intraperitoneally; Chemicon) was given 1 hour before and 4 hours after CSD induction. GM6001 was administered intraperitoneally as a suspension of $7 \mathrm{mg} / \mathrm{ml}$ in $45 \%$ cyclodextrin (weight/volume) as described (26). Controls received the same volume of vehicle ( $45 \%$ cyclodextrin [weight/volume]) 1 hour before and 4 hours after three CSDs.

To ensure that CSD and not needle stab or traumatic injury activated MMPs, the NMDA receptor blocker MK-801 (Sigma-Aldrich) was applied topically $(5 \mathrm{mM} ; 100 \mu \mathrm{l}) 15$ minutes before the needle stab. MK-801 blocks CSD wave propagation but does not block MMP enzymatic activation directly (our unpublished data).

Albumin leakage and brain edema measurements. For quantification of tracer after CSD, 2\% Evans blue was injected (3- $\mathrm{ml} / \mathrm{kg}$ dose via the tail vein 15 minutes before CSD induction). Rats were perfused with cold saline, and tissues from piriform or other homologous cortices were weighted and homogenized in a ten- fold volume of $50 \%$ trichloroacetic acid. After centrifugation, the supernatants were diluted fourfold with ethanol. Fluorescence intensity was measured by a microplate fluorescence reader (620 $\mathrm{nm}$ excitation, $680 \mathrm{~nm}$ emission; Wallac, Perkin Elmer, Wellesley, Massachusetts, USA). Calculations were based on external standard readings and extravasated dye was expressed as nanograms per milligram tissue or as leakage on the CSD/non-CSD sides. Edema was expressed as dry weight/wet weight of CSD brain tissue. After CSD, brains were removed and weighed, then were dried at $99^{\circ} \mathrm{C}$ for 48 hours and reweighed.

Statistical analysis. All data are presented as mean \pm SEM values. Differences between groups were determined by Student's $t$ test or by two-way ANOVA for repeated measures or one-way ANOVA followed by the Bonferroni correction. $P$ values less than 0.05 were considered statistically significant.

\section{Acknowledgments}

This work was funded by the NIH-sponsored Stroke Program Project (NS10828). Y. Gursoy-Ozdemir was supported partly by an International Brain Research Organization fellowship and a Fulbright scholarship. G. Rosenberg was supported by NIH R01 NS21169-11 and an American Heart Association Grant-in-Aid. We thank Christian Waeber and Munire Kilinc for their support and for their excellent technical assistance. We want to thank Robert Senior (Washington University, St Louis) for the use of MMP-9 knockout mice.

Received for publication February 3, 2004, and accepted in revised form March 23, 2004.

Address correspondence to: Michael A. Moskowitz, Massachusetts General Hospital, Stroke and Neurovascular Regulation Laboratory, 149 13th Street, Room 6403, Charlestown, Massachusetts 02129, USA. Phone: (617) 726-8440; Fax: (617) 726-2547; E-mail: moskowitz@helix.mgh.harvard.edu.

Yasemin Gursoy-Ozdemir and Jianhua Qiu contributed equally to this work.
1. Sugaya, E., Takato, M., and Noda, Y. 1975 Neuronal and glial activity during spreading depression in cerebral cortex of cat. J. Neurophysiol. 38:822-841.

2. Kager, H., Wadman, W.J., and Somjen, G.G. 2000. Simulated seizures and spreading depression in a neuron model incorporating interstitial space and ion concentrations. J. Neurophysiol. 84:495-512.

3. Leao, A.A.P. 1944. Pial circulation and spreading depression activity in cerebral cortex. J. Neurophysiol. 7:391-396

4. Pantoni, L., Lamassa, M., and Inzitari, D. 2000. Transient global amnesia: a review emphasizing pathogenic aspects. Acta Neurol. Scand. 102:275-283.

5. Martins-Ferreira, H., Nedergaard, M., and Nicholson, C. 2000. Perspectives on spreading depression. Brain Res. Brain Res. Rev. 32:215-234.

6. Otori T., Greenberg, J.H., and Welsh, F.A. 2003. Cortical spreading depression causes a long-lasting decrease in cerebral blood flow and induces tolerance to permanent focal ischemia in rat brain. J. Cereb. Blood Flow Metab. 23:43-50.

7. Jander, S., Schroeter, M., Peters, O., Witte, O.W., and Stoll, G. 2001. Cortical spreading depression induces proinflammatory cytokine gene expression in the rat brain. J. Cereb. Blood Flow Metab. 21:218-225.

8. Nedergaard, M., and Hansen, A.J. 1988. Spreading depression is not associated with neuronal injury in the normal brain. Brain Res. 24; 449:395-398.

9. Yong, V.W., Krekoski, C.A., Forsyth, P.A., Bell, R., and Edwards, D.R. 1998. Matrix metalloproteinases and diseases of the CNS. Trends Neurosci. 21:75-80.

10. Yong, V.W., Power, C., Forsyth, P., and Edwards, D.R. 2001. Metalloproteinases in biology and pathology of the nervous system. Nat. Rev. Neurosci. 2:502-511.

11. Rosenberg, G.A. 2002. Matrix metalloproteinases in neuroinflammation. Glia. 39:279-291.

12. Chandler, S., et al. 1997 .Matrix metalloproteinases, tumor necrosis factor and multiple sclerosis: an overview. J. Neuroimmunol. 72:155-161.

13. Aoki, T., Sumii, T., Mori, T., Wang, X., and Lo, E.H. 2002. Blood-brain barrier disruption and matrix metalloproteinase- 9 expression during reperfusion injury: mechanical versus embolic focal ischemia in spontaneously hypertensive rats. Stroke. 33:2711-2717.

14. Leppert, D., et al. 2000. Matrix metalloproteinase (MMP)-8 and MMP-9 in cerebrospinal fluid during bacterial meningitis: association with bloodbrain barrier damage and neurological sequelae. Clin. Infect. Dis. 31:80-84.

15. Rosenberg, G.A., et al. 1992. TIMP-2 reduces proteolytic opening of blood-brain barrier by type IV collagenase. Brain Res. 576:203-207.

16. Leppert, D., Waubant, E., Galardy, R., Bunnett,
N.W., and Hauser, S.L. 1995. T cell gelatinases mediate basement membrane transmigration in vitro. J. Immunol. 154:4379-4389.

17. Créange, A., et al. 1999. Matrix metalloproteinase9 is increased and correlates with severity in Guillain-Barré syndrome. Neurology. 53:1683-1691.

18. Brinckerhoff, C.E., and Matrisian, L.M. 2002. Matrix metalloproteinases: a tail of a frog that became a prince. Nat. Rev. Mol. Cell Biol. 3:207-214.

19. Asahi, M., et al. 2000. Role for matrix metalloproteinase 9 after focal cerebral ischemia: effects of gene knockout and enzyme inhibition with BB-94. J. Cereb. Blood Flow Metab. 20:1681-1689.

20. Asahi, M., et al. 2001. Effects of matrix metalloproteinase-9 gene knock-out on the proteolysis of blood-brain barrier and white matter components after cerebral ischemia. J. Neurosci. 21:7724-7732.

21. Rosenberg, G.A., et al. 2001. Immunohistochemistry of matrix metalloproteinases in reperfusion injury to rat brain: activation of MMP-9 linked to stromelysin-1 and microglia in cell cultures. Brain Res. 893:104-112.

22. Gasche, Y., et al. 1999. Early appearance of activated matrix metalloproteinase-9 after focal cerebral ischemia in mice: a possible role in blood-brain barrier dysfunction. J. Cereb. Blood Flow Metab. 19:1020-1028.

23. Lo, E.H., Wang, X., and Cuzner, M.L. 2002. Extracellular proteolysis in brain injury and 
inflammation: role for plasminogen activators and matrix metalloproteinases. J. Neurosci. Res. 69:1-9.

24. Bolay, H., et al. 2002. Intrinsic brain activity triggers trigeminal meningeal afferents in a migraine model. Nat. Med. 8:136-142.

25. Leib, S.L., Leppert, D., Clements, J., and Täuber, M.G. 2000. Matrix metalloproteinases contribute to brain damage in experimental pneumococcal meningitis. Infect. Immun. 68:615-620.

26. Szklarczyk, A., Lapinska, J., Rylski, M., McKay, R.D., and Kaczmarek, L. 2002. Matrix metalloproteinase- 9 undergoes expression and activation during dendritic remodeling in adult hippocampus. J. Neurosci. 22:920-930.

27. Hu, W., Kharlamov, A., Wang, Y., Perez-Trepichio, A.D., and Jones, S.C. 2000. Directed sampling for electrolyte analysis and water content of micropunch samples shows large differences between normal and ischemic rat brain cortex. Brain Res. 868:370-375

28. Paczynski, R.P., He, Y.Y., Diringer, M.N., and Hsu, C.Y. 1997. Multiple-dose mannitol reduces brain water content in a rat model of cortical infarction. Stroke. 28:1437-1444.

29. Hanemaaijer, R., Koolwijk, P., le Clercq, L., de Vree, W.J., and van Hinsbergh, V.W. 1993. Regulation of matrix metalloproteinase expression in human vein and microvascular endothelial cells. Effects of tumour necrosis factor alpha, interleukin 1 and phorbol ester. Biochem. J. 296:803-809.

30. Wang, X., et al. 2003. Lipoprotein receptor-mediated induction of matrix metalloproteinase by tissue plasminogen activator. Nat. Med. 9:1313-1317.

31. Rivera, S., et al. 2002. Gelatinase B and TIMP-1 are regulated in a cell- and time-dependent manner in association with neuronal death and glial reactivity after global forebrain ischemia. Eur. J. Neurosci. 15:19-32.

32. Vecil, G.G., et al. 2000. Interleukin-1 is a key regulator of matrix metalloproteinase- 9 expression in human neurons in culture and following mouse brain trauma in vivo. J. Neurosci. Res. 61:212-224.

33. Moon, S.K., Cha, B.Y., and Kim, C.H. 2003. In vitro cellular aging is associated with enhanced proliferative capacity, G1 cell cycle modulation, and matrix metalloproteinase- 9 regulation in mouse aortic smooth muscle cells. Arch. Biochem. Biophys. 418:39-48.

34. James, T.W., Wagner, R., White, L.A., Zwolak, R.M., and Brinckerhoff, C.E. 1993. Induction of collagenase and stromelysin gene expression by mechanical injury in a vascular smooth muscle-derived cell line. J. Cell Physiol. 157:426-437.

35. Fabunmi, R.P., Baker, A.H., Murray, E.J., Booth,
R.F., and Newby, A.C. 1996. Divergent regulation by growth factors and cytokines of $95 \mathrm{kDa}$ and 72 $\mathrm{kDa}$ gelatinases and tissue inhibitors or metalloproteinases-1, -2 , and -3 in rabbit aortic smooth muscle cells. Biochem. J. 315:335-342.

36. Cho, A., and Reidy, M.A. 2002. Matrix metalloproteinase- 9 is necessary for the regulation of smooth muscle cell replication and migration after arterial injury. Circ. Res. 91:845-851.

37. Vaillant, C., Didier-Bazes, M., Hutter, A., Belin, M.F., and Thomasset, N. 1999. Spatiotemporal expression patterns of metalloproteinases and their inhibitors in the postnatal developing rat cerebellum. J. Neurosci. 19:4994-5004.

38. Vecil, G.G., et al. 2000. Interleukin-1 is a key regulator of matrix metalloproteinase- 9 expression in human neurons in culture and following mouse brain trauma in vivo. J. Neurosci. Res. 61:212-224.

39. Read, S.J., Smith, M.I., Hunter, A.J., and Parsons, A.A. 1997. The dynamics of nitric oxide release measured directly and in real time following repeated waves of cortical spreading depression in the anaesthetised cat. Neurosci. Lett. 232:127-130.

40. Obrenovitch, T.P., Urenjak, J., and Wang, M. 2002. Nitric oxide formation during cortical spreading depression is critical for rapid subsequent recovery of ionic homeostasis. J. Cereb. Blood Flow Metab. 22:680-688.

41. Gu, Z., et al. 2002. S-nitrosylation of matrix metalloproteinases: signaling pathway to neuronal cell death. Science. 297:1186-1190.

42. Gasche, Y., Copin, J.C., Sugawara, T., Fujimura, M., and Chan, P.H. 2001. Matrix metalloproteinase inhibition prevents oxidative stress-associated blood-brain barrier disruption after transient focal cerebral ischemia. J. Cereb. Blood Flow Metab. 21:1393-1400.

43. Kim, G.W., et al. 2003. Neurodegeneration in striatum induced by the mitochondrial toxin 3 nitropropionic acid: role of matrix metalloproteinase- 9 in early blood-brain barrier disruption? J. Neurosci. 23:8733-8742.

44. von Gertten, C., Holmin, S., Mathiesen, T., and Nordqvist, A.C. 2003. Increases in matrix metalloproteinase- 9 and tissue inhibitor of matrix metalloproteinase-1 mRNA after cerebral contusion and depolarization. J. Neurosci. Res. 73:803-810.

45. Hamann, G.F., Okada, Y., Fitridge, R., and del Zoppo, G.J. 1995. Microvascular basal lamina antigens disappear during cerebral ischemia and reperfusion. Stroke. 26:2120-2126.

46. Ghabriel, M.N., Zhu, C., and Leigh, C. 2002. Electron microscope study of blood-brain barrier opening induced by immunological target- ing of the endothelial barrier antigen. Brain Res. 934:140-151.

47. Lin, B., et al. 2001. Quantitative analysis of microvascular alterations in traumatic brain injury by endothelial barrier antigen immunohistochemistry. J. Neurotrauma. 18:389-397.

48. Lin, B., and Ginsberg, M.D. 2000. Quantitative assessment of the normal cerebral microvasculature by endothelial barrier antigen (EBA) immunohistochemistry: application to focal cerebral ischemia. Brain Res. 865:237-244.

49. Kakinuma, Y., et al. 1998. Impaired blood-brain barrier function in angiotensinogen-deficient mice. Nat. Med. 4:1078-1080.

50. Gualandris, A., Jones, T.E., Strickland, S., and Tsirka, S.E. 1996. Membrane depolarization induces calcium-dependent secretion of tissue plasminogen activator. J. Neurosci. 16:2220-2225.

51. Nedergaard, M., and Hansen A.J. 1993. Characterization of cortical depolarizations evoked in focal cerebral ischemia. J. Cereb. Blood Flow Metab. 13:568-574.

52. Back, T., Kohno, K., and Hossmann, K.A. 1994. Cortical negative DC deflections following middle cerebral artery occlusion and $\mathrm{KCl}$-induced spreading depression: effect on blood flow, tissue oxygenation, and electroencephalogram. J. Cereb. Blood Flow Metab. 14:12-19.

53. Ohta, K., Graf, R., Rosner, G., and Heiss, W.D. 2001. Calcium ion transients in peri-infarct depolarizations may deteriorate ion homeostasis and expand infarction in focal cerebral ischemia in cats. Stroke. 32:535-543.

54. Back, T., Ginsberg, M.D., Dietrich, W.D., and Watson, B.D. 1996. Induction of spreading depression in the ischemic hemisphere following experimental middle cerebral artery occlusion: effect on infarct morphology. J. Cereb. Blood Flow Metab. 16:202-213.

55. Kruit, M.C., et al. 2004. Migraine as a risk factor for subclinical brain lesions. JAMA. 291:427-434.

56. Dietrich, W.D., Feng, Z.C., Leistra, H., Watson, B.D., and Rosenthal, M. 1994. Photothrombotic infarction triggers multiple episodes of cortical spreading depression in distant brain regions. J. Cereb. Blood Flow Metab. 14:20-28.

57. Strong, A.J., et al. 2002. Spreading and synchronous depressions of cortical activity in acutely injured human brain. Stroke. 33:2738-2743.

58. Ayata, C., Shimizu-Sasamata, M., Lo, E.H., Noebels, J.L., and Moskowitz, M.A. 2000. Impaired neurotransmitter release and elevated threshold for cortical spreading depression in mice with mutations in the alpha1A subunit of P/Q type calcium channels. Neuroscience. 95:639-645. 